\title{
Providing Training Enhances the Biomechanical Improvements of an Alternative Computer Mouse Design
}

\author{
Annemieke Houwink and Karen M. Oude Hengel, VU University Amsterdam, \\ Netherlands, Dan Odell, Microsoft Corporation, Redmond, Washington, and \\ Jack T. Dennerlein, Harvard School of Public Health, Boston, Massachusetts
}

\begin{abstract}
Objective: The purpose of this study is to determine if an alternative mouse promotes more neutral postures and decreases forearm muscle activity and if training enhances these biomechanical benefits. Background: Computer mouse use is a risk factor for developing musculoskeletal disorders; alternative mouse designs can help lower these risks. Ergonomic training combined with alternative input devices could be even more effective than alternative designs alone. Methods: Thirty healthy adults (15 males, 15 females) performed a set of computer mouse tasks with a standard mouse and an alternative mouse while an electromagnetic motion analysis system measured their wrist and forearm postures and surface electromyography measured the muscle activity of three wrist extensor muscles. Fifteen participants received no training on how to hold the alternative mouse, whereas the remaining 15 participants received verbal instructions before and during use of the alternative mouse. Results: The alternative mouse was found to promote a more neutral forearm posture compared with the standard mouse (up to $11.5^{\circ}$ lower forearm pronation); however, pronation was further reduced when instructions on how to hold the mouse were provided. Wrist extensor muscle activity was reduced for the alternative mouse (up to $1.8 \%$ of maximum voluntary contraction lower) compared with the standard mouse, but only after participants received instructions. Conclusion: The alternative mouse design decreased biomechanical exposures; however, instructions enhanced this potential ergonomic benefit of the design. Application: User knowledge and training are important factors when effectively implementing an alternative ergonomic device.
\end{abstract}

\section{INTRODUCTION}

More than half of all employees in the United States used a computer in 2003 (Bureau of Labor Statistics, 2005). The computer mouse has become an essential part of computer work, as $30 \%$ to $80 \%$ of computer work involves the mouse (Dennerlein \& Johnson, 2006). In addition, duration of mouse use is highly associated with upper extremity and neck musculoskeletal symptoms and disorders (Cook, BurgessLimerick, \& Chang, 2000; IJmker et al., 2007; Jensen, Finsen, Søgaard, \& Christensen, 2002).

Biomechanical risk factors include nonneutral postures, specifically, extreme pronation (Rempel, Bach, Gordon, \& So, 1998), radial deviation (Marcus et al., 2002), ulnar deviation, and wrist extension (Jensen et al., 1998;
Karlqvist, Hagberg, \& Selin, 1994). These nonneutral postures are observed during mouse use (Burgess-Limerick, Schemmell, Scadden, \& Plooy, 1999; Jensen et al., 1998) and are associated with higher muscle activity (Fagarasanu, Kumar, \& Narayan, 2004). Sustained muscle activity is also thought to be a great risk factor for muscle fiber disturbances and damage (Gissel, 2000; Hägg, 2000), which is characteristic of computer work and mouse use (Sjøgaard \& Søgaard, 1998).

Alternative input devices are often used as an intervention to reduce the risk of developing musculoskeletal symptoms or musculoskeletal disorders (Conlon, Krause, \& Rempel, 2007; Rempel et al., 2006). Alternative input devices that promote a more neutral forearm posture (less pronation) are found to reduce pain in 
TABLE 1: Demography and Anthropometry for the Untrained Group and the Trained Group With Standard Deviation (in parentheses) and Level of Significance ( $p$ value)

\begin{tabular}{lcccccccc}
\hline & \multicolumn{2}{c}{ Persons } & \multicolumn{2}{c}{ Age (years) } & \multicolumn{2}{c}{ Hand length $(\mathrm{cm})$} & \multicolumn{2}{c}{ Hand width (cm) } \\
\hline Group & Total & Male & Mean & Range & Mean & Range & Mean & Range \\
\hline Untrained & 15 & 7 & $28.7(5.6)$ & $(23-43)$ & $17.7(1.3)$ & $(16.0-19.5)$ & $8.2(0.6)$ & $(7.5-9.6)$ \\
Trained & 15 & 8 & $31.5(11.1)$ & $(19-58)$ & $18.7(1.5)$ & $(16.0-21.0)$ & $8.3(0.8)$ & $(7.0-9.5)$ \\
$p$ Value & & 0.58 & 0.40 & & 0.07 & & 0.77 & \\
\hline
\end{tabular}

the upper extremity (Aarås, Dainoff, Ro, \& Thoresen, 2001). Other studies found reduced forearm muscle activity and more neutral postures when participants used a computer mouse that promoted less forearm pronation (Chen \& Leung, 2007; Gustafsson \& Hagberg, 2003).

Another often-used intervention to prevent musculoskeletal disorders is ergonomic training, which, according to Van der Molen, Sluiter, Hulshof, Vink, and Frings-Dresen (2005), was more effective in reducing workload when implemented in ergonomic adjustments. Other previous studies found similar results: that ergonomic training combined with workplace adjustments had a greater effect than ergonomic training alone (Rempel et al., 2006) and that workplace adjustments (a highly adjustable chair) in combination with ergonomic training reduced the development of musculoskeletal symptoms during the workday, which was more effective than workplace adjustments alone (Amick et al., 2003).

Although benefits of alternative mouse designs and ergonomic training separately have been found, the effects of training on the use of an alternative mouse are not clear. Therefore, this study aims to determine if an alternative mouse design promotes more neutral postures and decreases muscle activity and if training enhances the biomechanical benefits. In a repeated-measures laboratory experiment, posture and muscle activity were measured to compare biomechanical exposure between an alternative mouse and a standard mouse.

\section{METHODS AND MATERIAL}

\section{Participants}

Thirty healthy right-handed adults (15 males and 15 females) participated in this study (Table 1).
All participants were experienced in working with computer mice (mean self-reported computer time weekly $=38 \mathrm{hr}$ ). Participants were divided into two equal-sized groups and either did or did not receive training. There were no significant differences in anthropometry measures between groups (Table 1). All participants provided signed consent, and all experimental procedures were approved by the Harvard School of Public Health Human Subjects Committee.

\section{Experimental Protocol}

The participants performed a standard set of pointing tasks with two commercially available computer mice while muscle activity and posture of the forearm and hand were measured. One was a symmetrical standard mouse (Microsoft Wireless Optical Mouse 2000) and the other, an asymmetrical alternative mouse that was tilted to promote less forearm pronation (Microsoft Natural Wireless Laser Mouse 6000) (Figure 1).

For all tasks, participants were seated at a workstation that consisted of a chair with no armrests, a work surface, and a flat-panel monitor on a monitor stand. All the components were adjusted to each individual such that the participant's thighs were horizontal to the ground and the table surface was at resting elbow height. The keyboard was centered with the participant and its position marked on the work surface. For each experimental condition, the mouse was positioned to the right of the keyboard. For all mice, conditions, and participants, the pointer speed within the mouse device control panel remained constant.

Before starting with the standard mouse, participants practiced with the mouse while being instructed to hold and use the mouse as they thought they should when looking at the design. 


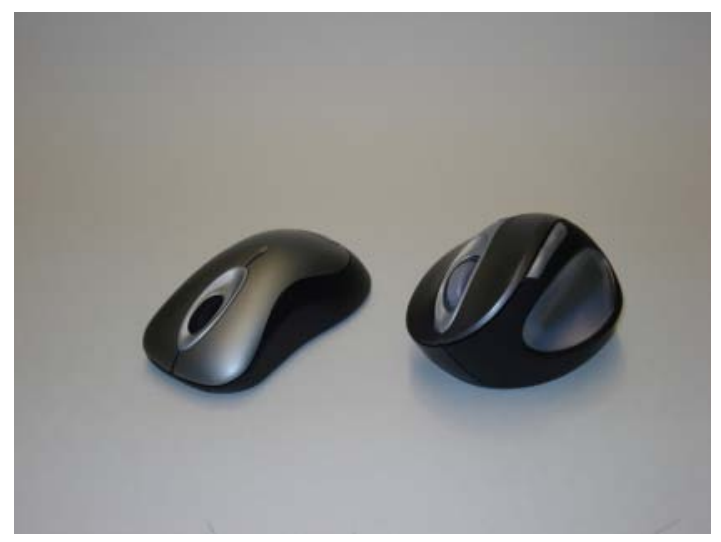

Figure 1. Frontal-lateral view of the standard mouse (left) and alternative mouse (right).

The untrained group members received the same instructions for the alternative mouse and were allowed to practice until they acknowledged that they were comfortable using the mouse. The trained group received instructions on the use of the alternative mouse before starting the tasks. This training consisted of oral instructions and coaching on how to hold the alternative mouse, which involved placing the hand on the table just next to the mouse with the thumb pointing upward and then pronating the forearm to grip the mouse. The participants practiced the pointing tasks (which are described later) for 5 to 10 targets with the alternative mouse after receiving these instructions. During the practice session, the verbal instructions were repeated if the participants in the trained group did not follow the instructions and held the alternative mouse in a manner not instructed. Because a different experimental protocol was completed on the same set of participants (Oude Hengel, Houwink, Odell, Van Dieën, \& Dennerlein, 2008), randomization of the mice was not possible, and each participant had to use the standard mouse first and then the alternative mouse.

With each mouse, participants completed four standard mouse tasks: a large-sized pointand-click task, a small-sized point-and-click task, a dragging task, and a steering task. Each task was omnidirectional and lasted approximately $1 \mathrm{~min}$. The large-sized and small-sized point-and-click tasks and the dragging task consisted of evenly spaced target circles arranged in a large circle with a radius of $120 \mathrm{~mm}$
(356 pixels). The target circles of the large-sized point-and-click task and the dragging task had a radius of $7 \mathrm{~mm}$ (21 pixels), whereas the target circles of the small-sized point-and-click tasks had a radius of $2 \mathrm{~mm}$ (6 pixels). For the largesized and small-sized point-and-click tasks, participants had to click on an active circle; for the dragging task, they had to select (press the button) an active circle and drag it to the opposite side of the large circle (release the button). For the steering task, participants had to select a small target circle (radius of $3 \mathrm{~mm}$, or 9 pixels) and then move the circle from one point to another while maintaining its position within the boundaries of a tunnel $(20 \mathrm{~mm}$, or 59 pixels, wide and $120 \mathrm{~mm}$, or 356 pixels, long).

All tasks were presented via a customdeveloped software program in LabView (National Instruments, Austin, TX) on a monitor set to $1,024 \times 768$ pixels. To measure performance, the program recorded the time to complete each task. Participants were told before the tasks to work as quickly as possible while remaining accurate.

\section{Measuring Instruments}

During the experiment, surface electromyographic (EMG) electrodes (DE-2.1 Single Differential Electrode; Delsys, Boston, MA) measured muscle activity for three forearm muscles of the right arm. Electrodes were placed on the muscle bellies of the extensor carpi radialis (ECR), the extensor digitorum communis (EDC), and the extensor carpi ulnaris (ECU), as recommended by Perotto (1994). Placement of electrodes was validated through palpation and signal response to isometric test contractions. The EMG signals were amplified and band-pass filtered (20-450 $\mathrm{Hz}$, Bagnoli-eight amplifier; Delsys) before being digitally recorded at a sample rate of 1,000 samples per second.

To normalize the EMG results across participants, three 5-s maximum voluntary isometric contractions were collected for each muscle while the experimenter manually restrained the movement of the joint about which the muscle of interest articulated. One hand was placed most proximal to the joint to restrain compensatory movements while the other hand was placed 
most distal to the joint to block the requested movement. The experimenter applied force against the direction of the movement to enable the participant to generate maximum force. The requested movement directions for the muscles were wrist extension combined with radial deviation for the ECR, wrist extension combined with ulnar deviation for the ECU, and extension of the fingers for the EDC. Participants rested between contractions for $1 \mathrm{~min}$. During the maximum voluntary contractions, participants were seated with their elbows flexed $\left(90^{\circ}\right)$ and their forearm fully pronated, simulating the general posture during the experiments.

An electromagnetic motion analysis system (Minibird; Ascension Technology, Burlington, VT) measured the position and orientation of the forearm and hand of the right arm at a sample rate of 20 samples/s. Two sensors $(12 \times 7 \times 7 \mathrm{~mm})$ were placed on the dorsal sides of the metacarpus (midway on the third metacarpal bone) and forearm (between ulna and radius, located one third of the distance from the wrist to the elbow). Before starting the data collection, we recorded a reference position of the hand and arm using a calibration fixture as described by P. Jonsson and Johnson (2001). The neutral postures of the wrist were then defined as the hand aligned with the forearm (the middle finger aligned with the middle of the forearm) with the palm of the hand flat on the calibration fixture and the forearm fully pronated. For the forearm, this position was defined as $90^{\circ}$ pronation; therefore, the neutral posture was defined as the forearm and hand $90^{\circ}$ rotated from full pronation, such that the thumb was pointing upward.

\section{Data Processing and Statistical Analysis}

The EMG amplitude was represented by a root mean square value calculated from the raw data over a 0.2 -s moving window and then normalized by the root mean square value obtained during the maximum voluntary contractions. The maximum voluntary contraction value was the highest root mean square amplitude averaged from three maximum voluntary contractions. Relative joint angles of the wrist and forearm were calculated from the orientations of the hand and forearm Minibird sensors.

For each participant and mouse, summary statistics were calculated for posture, muscle activity, and task performance. The first and last $10 \mathrm{~s}$ of each task were removed to ensure that the data contained actual mouse work. The summary statistics included the 10th, 50th, and 90th percentiles of the EMG amplitude and the relative joint angles. For the EMG values, B. Jonsson (1988) defined the 10th percentile as the static component of muscle load and the 50th and 90th percentiles as the dynamic components. For the postural data, the 10th and 90th percentiles represented postural extremes, and the difference between the 90th and the 10th percentile represented the joint's range of motion. For performance, the average movement time was calculated, which was defined as the average time between each successful click, drag, and tunnel steer.

Differences in the EMG and postural measures between the two mice were analyzed using a repeated-measures ANOVA. To determine differences between mice for the trained and untrained group, a between-subjects nesting factor was added to the repeated-measures ANOVA. A one-way ANOVA was used to analyze differences within each mouse. All statistics were performed using SPSS 13.0. Significance was noted for a probability of a false positive being less than $5 \%(p<.05)$.

\section{RESULTS}

Postures differed between the alternative and standard mouse for the untrained and trained groups (Table 2). For one participant in the trained group, data were not recorded properly; therefore, this group consisted of 14 participants.

Forearm pronation for both groups was lower with the alternative mouse than with the standard mouse $(p<.001$; Figure 2$)$; however, lower pronation was measured for the trained group than for the untrained group (up to $11.5^{\circ}$ and $6.5^{\circ}$ lower, respectively; Table 2). For wrist extension, participants in both groups had higher wrist extension with the alternative mouse than with the standard mouse; however, differences between the alternative and the standard mouse for the untrained group were larger (up to $5.6^{\circ}$ ) than for the trained group (up to $1.8^{\circ}$; Figure 2). The differences in wrist extension and forearm pronation between the trained and the untrained 
TABLE 2: Postural Values in Degrees (10th, 50th, 90th Percentiles and Range of Motion [ROM]) With Standard Errors (in parentheses) for Right Wrist and Forearm

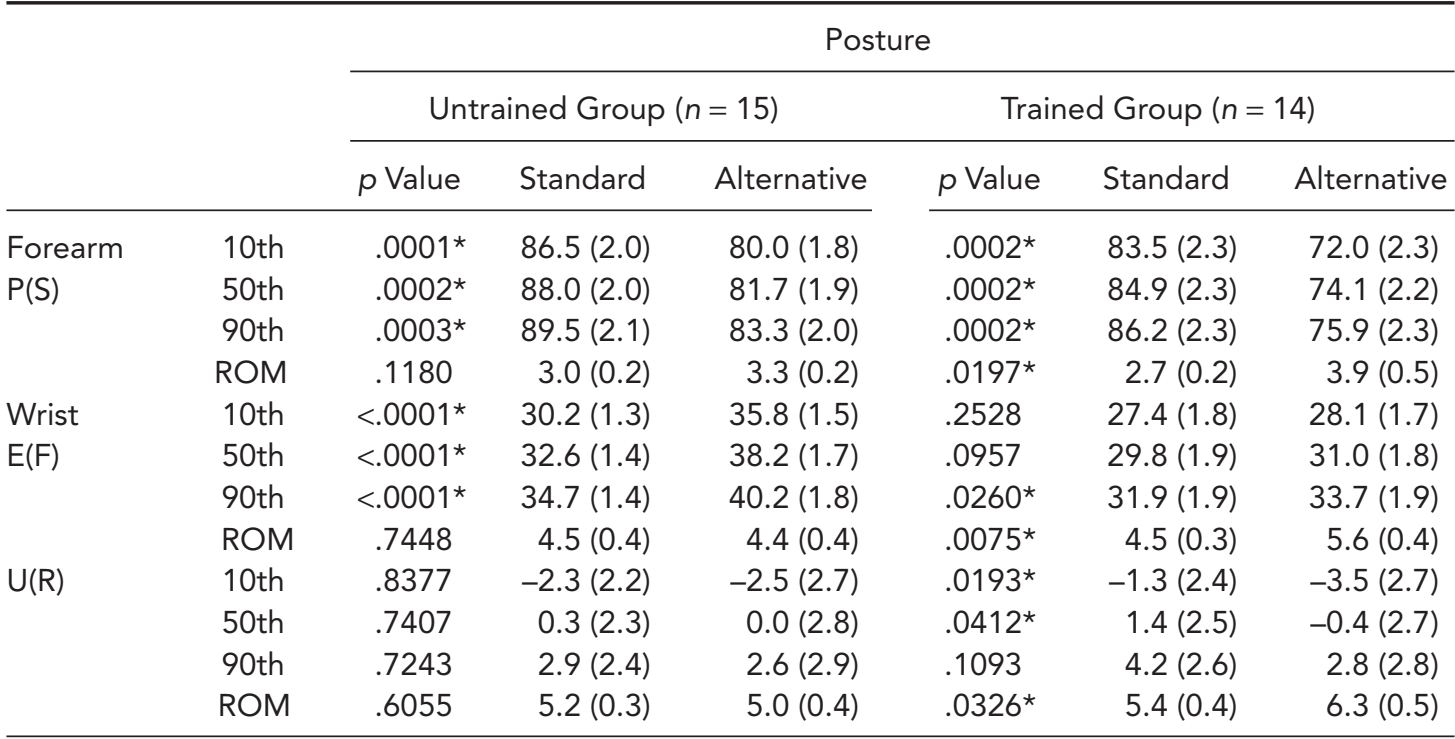

Note. Positive values indicate pronation for the forearm, and extension and ulnar deviation for the wrist.

$P(S)=$ pronation (supination); $E(F)=$ extension (flexion); $U(R)=$ ulnar (radial) deviation.

* denotes significant differences between standard and alternative mouse within untrained or trained group.

group were significant with the alternative mouse $(p<.05)$ but not with the standard mouse (Figure 2). For wrist deviation, no differences between the mice were found for the untrained group. The trained group, however, had less ulnar deviation with the alternative mouse for the 10th and 50th percentiles $(p<.05)$ than with the standard mouse, which resulted in a radialoriented wrist posture. For the trained group, range of motion of wrist and forearm was higher with the alternative mouse than with the standard mouse $(p<.05)$; however, there was no significant difference in range of motion for the untrained group.

Muscle activities differed between mice for the trained group but not for the untrained group (Table 3). For the trained group, EMG values for all wrist extensors with the alternative mouse were lower than with the standard mouse $(p<.05)$. The differences between the untrained and the trained group were present only with the alternative mouse for the ECR and EDC activity $(p<.05)$ and not with the standard mouse. For the ECR muscle, the first 9 participants in the untrained group were not entered in the analysis because interference was observed that was larger than the EMG signal.
Performance, defined as movement time and averaged across all tasks, was slower when participants used the alternative mouse $(1.29 \mathrm{~s})$ than the standard mouse $(1.18 \mathrm{~s})$ in the untrained group $(p<.03)$. However, performance did not differ between the alternative mouse $(1.27 \mathrm{~s})$ and the standard mouse $(1.22 \mathrm{~s})$ in the trained group $(p=.229)$.

\section{DISCUSSION}

The alternative mouse design used in this study promoted a more neutral forearm posture through its shape and the location of the buttons and the thumb and finger wells. In general, users realized and utilized this benefit; however, this effect was enhanced by providing specific instructions on how to hold the mouse (Figure 3). In contrast, forearm muscle activity did not improve for all users; muscle activity decreased only after they received instructions. This indicates the importance of providing training when using an alternative mouse.

Previous studies on alternative mice found similar results when participants used a mouse that promoted a more neutral forearm posture. Gustafsson and Hagberg (2003) found 


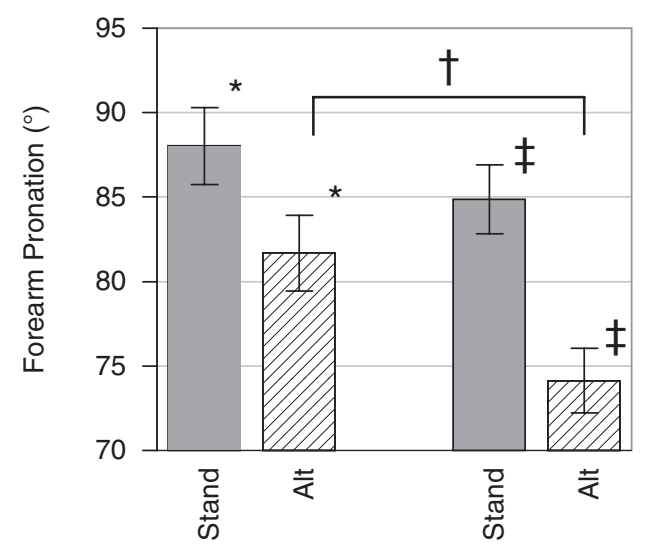

No training

Training

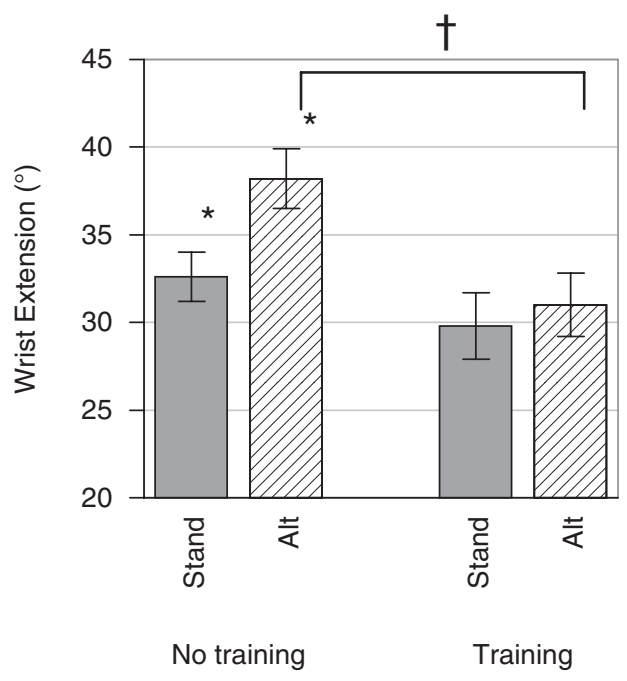

Figure 2. Forearm and wrist posture (median joint angle with standard error bars) differed between the standard (Stand) and the alternative (Alt) mouse and for the alternative mouse between the untrained (No training) and the trained group (Training). * denotes significant differences between the standard and the alternative mouse for the untrained group $(p<.001)$. $\dagger$ denotes significant differences between untrained and trained group for the alternative mouse $(p<.05)$. * denotes significant differences between the standard and the alternative mouse for the trained group $(p=.0002)$.

less wrist ulnar deviation and a trend toward less wrist extension when using a mouse with a neutral hand position ( $0^{\circ}$ pronation). Keir, Bach, and Rempel (1999) found similar values
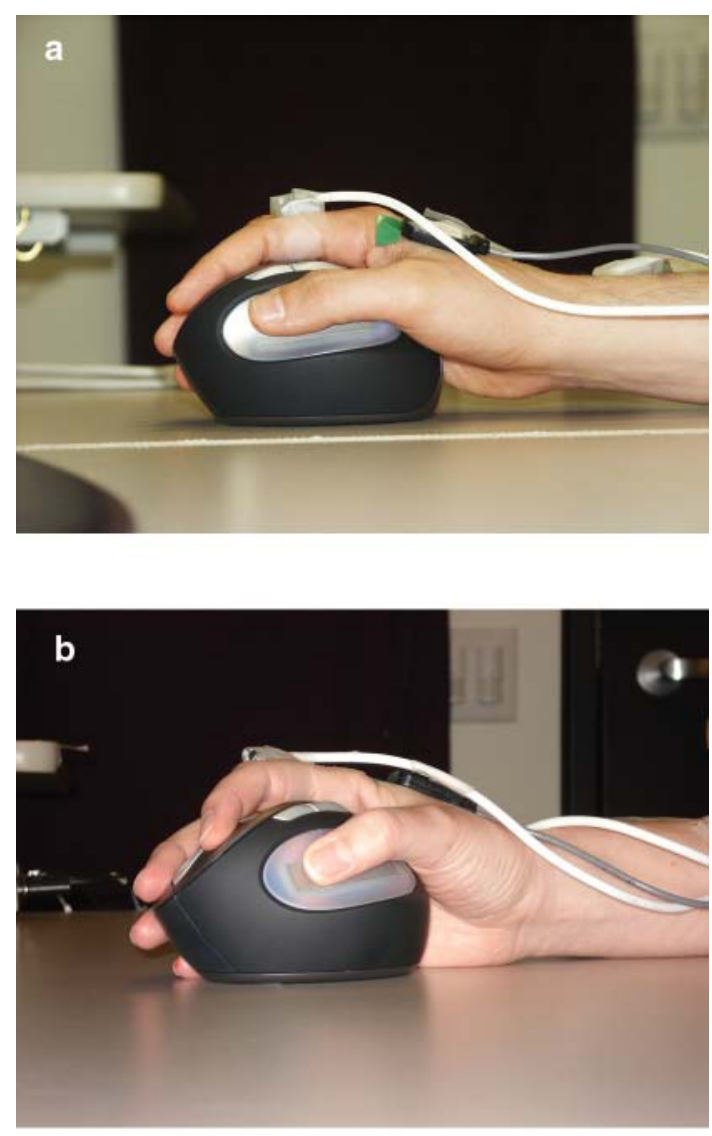

Figure 3. Hand positioning on the mouse for a participant who received no instructions (above) and for a participant who did receive instructions (below). With instructions, participants rested the ulnar side of their hands on the work surface, therefore decreasing wrist extension and forearm pronation.

of wrist extension when using different mice and an increase in carpal tunnel pressure during mouse tasks. Overall, carpal tunnel pressure increases with extension, and prolonged extension greater than $40^{\circ}$ can create pressures above $30 \mathrm{mmHg}$ (Keir, Bach, Hudes, \& Rempel, 2007).

In the present study, wrist extension was found to be similar with the alternative mouse and the standard mouse in the trained group but increased with no training. This indicates the importance of training and a mouse design that promotes less wrist extension, as high wrist extension may increase carpal tunnel pressure, which can create physiological nerve damage in 
TABLE 3: The 10th, 50th, and 90th Percentile Electromyography (EMG) Muscle Activity Values With Standard Errors (in parentheses)

\begin{tabular}{|c|c|c|c|c|c|c|c|}
\hline & \multirow[b]{3}{*}{ Percentile } & \multicolumn{6}{|c|}{ EMG } \\
\hline & & \multicolumn{3}{|c|}{ Untrained Group ( $n=15)$} & \multicolumn{3}{|c|}{ Trained Group $(n=14)$} \\
\hline & & $p$ Value & Standard & Alternative & $p$ Value & Standard & Alternative \\
\hline \multirow[t]{3}{*}{$\mathrm{ECR}^{\mathrm{a}}$} & 10th & 0.3381 & $4.5(0.8)$ & $4.7(0.8)$ & $0.0019 *$ & $3.3(0.4)$ & $2.9(0.4)$ \\
\hline & 50th & 0.2358 & $5.7(0.8)$ & $6.0(0.9)$ & $0.0062^{*}$ & $4.2(0.6)$ & $3.8(0.5)$ \\
\hline & 90th & 0.1543 & $7.3(1.0)$ & $7.9(1.2)$ & $0.0434^{\star}$ & $5.4(0.8)$ & $4.9(0.7)$ \\
\hline \multirow[t]{3}{*}{ EDC } & 10th & 0.2035 & $10.6(0.9)$ & $11.0(0.9)$ & $0.0002^{*}$ & $7.9(0.7)$ & $6.2(0.8)$ \\
\hline & 50th & 0.1364 & $12.0(0.9)$ & $12.6(0.9)$ & $0.0003^{*}$ & $10.0(0.8)$ & $8.2(0.9)$ \\
\hline & 90th & 0.1042 & $13.6(0.9)$ & $14.6(1.0)$ & $0.0020 *$ & $12.5(0.9)$ & $10.7(1.1)$ \\
\hline \multirow[t]{3}{*}{ ECU } & 10th & 0.5966 & $7.1(1.0)$ & $7.3(1.0)$ & $0.0102^{\star}$ & $7.7(0.9)$ & $6.0(0.6)$ \\
\hline & 50th & 0.3852 & $10.6(1.5)$ & $11.1(1.4)$ & $0.0483^{*}$ & $11.3(1.1)$ & $9.8(1.0)$ \\
\hline & 90th & 0.3988 & $15.7(2.1)$ & $16.4(2.1)$ & 0.1173 & $15.7(1.3)$ & $14.0(1.4)$ \\
\hline
\end{tabular}

Note. All values are in percentage of maximum voluntary contraction. $E C R=$ extensor carpi radialis; $E D C=$ extensor digitorum communis; ECU = extensor carpi ulnaris.

${ }^{a} \mathrm{~N}=6$ for the untrained group because of signal interference for the ECR muscle for the first 9 participants.

* Denotes significant differences between the standard and the alternative mouse within the untrained or the trained group.

the carpal tunnel (Rempel, Dahlin, \& Lundborg, 1999). Gustafsson and Hagberg (2003) also found a more radial-deviated position with the neutral mouse, which is similar to the findings in the present study, in which participants who received instructions had a smaller increase in radial deviation when using the alternative mouse than when using the standard mouse.

Although these differences in wrist posture were small in terms of absolute numbers, the impact of these changes during actual work over longer periods with the pressures and demands of a real-world job is still unknown. The deviation values were within the neutral range of wrist deviation ( -5 to $5^{\circ}$ of ulnar deviation), as suggested by Marcus et al. (2002). Although we observed some changes in performance in the untrained group, the changes were very small, and hence their relevance is unclear.

The reduction in EMG values for the wrist extensors with the alternative mouse in the trained group was consistent with findings by Chen and Leung (2007) and Gustafsson and Hagberg (2003). Both studies found a decrease in ECU activity when using an alternative mouse that promoted less pronation. The reduction in EDC activity found in the present study was also found by Gustafsson and Hagberg but not by Chen and Leung. Instructions on how to use a mouse could have influenced this difference, as muscle activity for the extensor muscles was not reduced for the untrained group. It was not stated in the other studies whether instructions were provided. ECR activity in the present study decreased when participants used the alternative mouse with instructions, but no previous studies measured this muscle activity, so no comparisons could be made.

The lower EMG values were related to the more neutral postures when participants used the alternative mouse in the trained group, which was also found by Fagarasanu et al. (2004). Although range of motion of the wrist and forearm was higher, muscle activity of the extensor muscles was lower. This difference could indicate that during alternative mouse use, participants used different muscles to compensate for the increase in range of motion. It was observed during the experiment that participants used more elbow and shoulder movements when using the alternative mouse with instructions. In this way, muscle activity of the extensor muscles would have remained the same, but other muscles would have been used more. The 
use of different muscles during mouse work could help lessen the burden of wrist extensor muscles, which are often affected by musculoskeletal disorders. However, the extra burden on other muscles should be investigated further, as discomfort in the shoulder-neck region is also reported by computer workers, although risk estimates are lower for the shoulder-neck region than for the hand-arm region (IJmker et al., 2007).

The results of the present study showed beneficial effects of providing ergonomic instructions on the use of an alternative mouse. Previous studies showed various results from ergonomic training. Amick et al. (2003) found that the risk of musculoskeletal symptoms was reduced more when combining ergonomic training with workplace adjustments (adjustable chair) than with workplace adjustments alone. Rempel et al. (2006) also found more effects of ergonomic training in combination with a workplace adjustment (forearm support) than with only ergonomic training. These results indicate that training or ergonomic adjustments alone are not enough to potentially prevent musculoskeletal disorders but that a combination of ergonomic training and workplace adjustments appears to be more beneficial.

The content of ergonomic training varied in the previous studies. Rempel et al. (2006), for instance, provided conventional recommendations on posture and workplace adjustments. Amick et al. (2003) used a different approach, which consisted of additional teaching (e.g., recognizing risk factors and understanding the importance of the ergonomic adjustments). Both studies found positive effects of combining training with workplace adjustments.

For the present study, the training consisted of ergonomic instructions that were provided by the manufacturer in the packaging of the alternative mouse. Because previous field studies demonstrated that providing training as well as equipment is more effective than providing only the equipment, the manufacturer's instructions were delivered orally, and participants were coached on how to apply these instructions during the use of the mouse. Although few computer mouse users are likely to receive training during the use of a newly designed device, training in the field does exist (ergonomic teams, consultants, workplace evaluations) in which people are instructed on how to achieve the benefits of the workstation design by adjusting equipment to achieve the best personal fit. The specific training tested here was effective in improving posture and lowering muscle activity. The next question is, what means are effective in providing training; for example, written instructions, interactive feedback, or other means?

The conclusions of this study are limited to mouse use in a laboratory workstation. Participants practiced only a short time with the alternative mouse; it is not clear if longer use could have affected results. However, preliminary testing of the design found that users did not adapt to the alternative design during longer sessions lasting up to a couple of hours. Also, participants were experienced in working with a standard mouse, but none of them had worked with the alternative mouse before, and it might have taken them longer to get used to it.

Furthermore, the order in which the mice were used among participants was not randomized. The reason is that the experimental design for the current study was added to another study in which participants used the standard mouse randomly among several notebook mice (Oude Hengel et al., 2008). As a result, all participants used the standard mouse first and the alternative mouse last. This could have resulted in a learning effect of the tasks, which would have shown in better performance results for the alternative mouse. However, the opposite occurred; the alternative mouse had longer movement times than the standard mouse in the untrained group and similar scores with training. Despite these limitations, this study has made an important attempt to study the biomechanical effects of an alternative mouse design in combination with training.

In conclusion, the alternative mouse promoted a more neutral forearm posture (less pronation) among all participants, even more so when they received instructions on how to use the mouse. However, muscle activity decreased only when instructions were provided, indicating the importance of ergonomic training. Instructions seem to be necessary for participants to fully benefit from ergonomic improvements in this alternative mouse design when it is first introduced. 


\section{ACKNOWLEDGMENTS}

This work was partly supported by a gift from Microsoft Corporation. The authors thank Pete Johnson for his work on the development of the mouse task program. Many thanks go out to our colleagues at the Harvard Occupational Biomechanics Lab for their support and advice during the writing of this manuscript.

\section{REFERENCES}

Aarås, A., Dainoff, M., Ro, O., \& Thoresen, M. (2001). Can a more neutral position of the forearm when operating a computer mouse reduce the pain level for visual display unit operators? A prospective epidemiological intervention study: Part II. International Journal of Human-Computer Interaction, 13, 13-40.

Amick, B. C., Robertson, M. M., DeRango, K., Bazzani, L., Moore, A., Rooney, T., et al. (2003). Effect of office ergonomics intervention on reducing musculoskeletal symptoms. Spine, 28, 2706-2011.

Bureau of Labor Statistics. (2005). Computer and Internet use at work in 2003. Washington, DC: U.S. Department of Labor, Bureau of Labor Statistics.

Burgess-Limerick, R., Schemmell, J., Scadden, R., \& Plooy, A. (1999). Wrist posture during computer pointing device use. Clinical Biomechanics, 14, 280-286.

Chen, H. M., \& Leung, C. T. (2007). The effect on forearm and shoulder muscle activity in using different slanted computer mice. Clinical Biomechanics, 22, 518-523.

Conlon, C. F., Krause, N., \& Rempel, D. M. (2007). A randomized controlled trial evaluating an alternative mouse and forearm support on upper body discomfort and musculoskeletal disorders among engineers. Occupational and Environmental Medicine, 65, 311-318.

Cook, C., Burgess-Limerick, R., \& Chang, S. (2000). The prevalence of neck and upper extremity musculoskeletal symptoms in computer mouse users. International Journal of Industrial Ergonomics, 26, 347-356.

Dennerlein, J. T., \& Johnson, P. W. (2006). Different computer tasks affect the exposure of the upper extremity to biomechanical risk factors. Ergonomics, 49, 45-61.

Fagarasanu, M., Kumar, S., \& Narayan, Y. (2004). Measurement of angular wrist neutral zone and forearm muscle activity. Clinical Biomechanics, 19, 671-677.

Gissel, H. (2000). $\mathrm{Ca}^{2+}$ accumulation and cell damage in skeletal muscle during low frequency stimulation. European Journal of Applied Physiology, 83, 175-180.

Gustafsson, E., \& Hagberg, M. (2003). Computer mouse use in two different hand positions: Exposure, comfort, exertion and productivity. Applied Ergonomics, 34, 107-113.

Hägg, G. M. (2000). Human muscle fibre abnormalities related to occupational load. European Journal of Applied Physiology, $83,159-165$.

IJmker, S., Huysmans, M. A., Blatter, B. M., Van der Beek, A. J., Van Mechelen, W., \& Bongers, P. M. (2007). Should office workers spend fewer hours at their computer? A systematic review of the literature. Occupational and Environmental Medicine, 64, 211-222.

Jensen, C., Borg, V., Finsen, L., Hansen, K., Juul-Kristensen, B., \& Christensen, H. (1998). Job demands, muscle activity and musculoskeletal symptoms in relation to work with the computer mouse. Scandinavian Journal of Work Environment and Health, 24, 418-424.

Jensen, C., Finsen, L., Søgaard, K., \& Christensen, H. (2002). Musculoskeletal symptoms and duration of computer and mouse use. International Journal of Industrial Ergonomics, 30, 265-275.

Jonsson, B. (1988). The static load component in muscle work. European Journal of Applied Physiology, 57, 305-310.

Jonsson, P., \& Johnson, P. W. (2001). Comparison of measurement accuracy between two types of wrist goniometer systems. Applied Ergonomics, 32, 599-607.

Karlqvist, L., Hagberg, M., \& Selin, K. (1994). Variation in upper limb posture and movement during word processing with and without mouse use. Ergonomics, 37, 1261-1267.

Keir, P. J., Bach, J. M., \& Rempel, D. (1999). Effect of computer mouse design and task on carpal tunnel pressure. Ergonomics, 42, 1350-1360.

Keir, P. J., Bach J. M., Hudes, M., \& Rempel, D. M. (2007). Guidelines for wrist posture based on carpal tunnel pressure thresholds. Human Factors, 49, 88-99.

Marcus, M., Gerr, F., Monteilh, C., Ortiz, D. J., Gentry, E., Cohen, S., et al. (2002). A prospective study of computer users: II. Postural risk factors for musculoskeletal symptoms and disorders. American Journal of Industrial Medicine, 41, 236-249.

Oude Hengel, K. M., Houwink, A., Odell, D., Van Dieën, J. H., \& Dennerlein, J. T. (2008). Smaller external notebook mice have different effects on posture and muscle activity. Clinical Biomechanics, 23, 727-734.

Perotto, A. (1994). Anatomical guide for the electromyographer: The limbs and trunk (3rd ed.). Springfield, IL: Charles C Thomas.

Rempel, D., Bach, J. M., Gordon, L., \& So, Y. (1998). Effects of forearm pronation/supination on carpal tunnel pressure. Journal of Hand Surgery, 23, 38-42.

Rempel, D., Dahlin, L., \& Lundborg G. (1999). Pathophysiology of nerve compression syndromes: Response of peripheral nerves to loading. Journal of Bone and Joint Surgery, American Volume, 81, 1600-1610.

Rempel, D. M., Krause, N., Goldberg, R., Benner, D., Hudes, M., \& Goldner, G. U. (2006). A randomised controlled trial evaluating the effects of two workstation interventions on upper body pain and incident musculoskeletal disorders among computer operators. Occupational and Environmental Medicine, 63, 300-306.

Sjøgaard, G., \& Søgaard, K. (1998). Muscle injury in repetitive motion disorders. Clinical Orthopaedics and Related Research, $351,21-31$

Van der Molen, H. F., Sluiter, J. K., Hulshof, C. T., Vink, P., \& Frings-Dresen, M. H. (2005). Effectiveness of measures and implementation strategies in reducing physical work demands due to manual handling at work. Scandinavian Journal of Work Environment and Health, 31, 75-87.

Annemieke Houwink received her master's degree in human movement sciences at the VU University of Amsterdam in 2007. She is a Ph.D. student at the Radboud University Nijmegen Medical Centre in the Netherlands.

Karen M. Oude Hengel received her master's degree in human movement sciences at the VU University of Amsterdam in 2007. She is a Ph.D. 
student at TNO Quality of Life | Work and Employment in Hoofddorp, Netherlands.

Dan Odell is an ergonomist and user researcher at Microsoft Corporation as well as a director for the Office Ergonomics Research Committee (www .oerc.org). He obtained his Ph.D. in mechanical engineering at the University of California, Berkeley, in 2004.
Jack T. Dennerlein is an associate professor of ergonomics and safety in the School of Public Health at Harvard University. He obtained his Ph.D. in mechanical engineering from the University of California, Berkeley, in 1996.

Date received: September 20, 2007

Date accepted: October 31, 2008 